\title{
Editorial
}

\section{Advanced Optimization Techniques and Their Applications in Civil Engineering}

\author{
Tayfun Dede $\mathbb{D}^{1},{ }^{1}$ Moacir Kripka $\left(\mathbb{D},{ }^{2}\right.$ Vedat Toğan, ${ }^{1}$ Victor Yepes $\left(\mathbb{D},{ }^{3}\right.$ and Ravipudi V. Rao ${ }^{4}$ \\ ${ }^{1}$ Department of Civil Engineering, Karadeniz Technical University, 61080 Trabzon, Turkey \\ ${ }^{2}$ Department of Civil Engineering, Universidade de Passo Fundo, 99001-970 Passo Fundo, RS, Brazil \\ ${ }^{3}$ ICITECH, Department of Construction Engineering, Universitat Politècnica de València, Camino de Vera $s / n$, \\ 46022 Valencia, Spain \\ ${ }^{4}$ Department of Mechanical Engineering, Sardar Vallabhai National Institute of Technology, Gujarat 395007, India
}

Correspondence should be addressed to Tayfun Dede; tayfundede@gmail.com

Received 17 May 2018; Accepted 19 May 2018; Published 25 June 2018

Copyright (C) 2018 Tayfun Dede et al. This is an open access article distributed under the Creative Commons Attribution License, which permits unrestricted use, distribution, and reproduction in any medium, provided the original work is properly cited.

Nowadays, a rapid growth of computer performance enables and encourages new developments in civil engineering as well as related areas. For instance, the construction industry investigates new designs with minimum cost, minimum $\mathrm{CO}_{2}$ emissions, or embodied energy, among other objectives. Applications of optimization techniques are most exciting, challenging, and of truly large scale when it comes to the problems of civil engineering in terms of both quality and quantity. In order to overcome the difficulties, researchers are interested in advanced optimization techniques. The aim of this special issue is to collect the studies using optimization algorithms in civil engineering problems such as structural engineering, construction management, and environmental engineering.

During the call for submissions, many papers were received and some of them were later withdrawn or rejected. The other 6 papers were accepted. The details about the published papers are given below.

The paper written by A. Saha, A. K. Saha, and S. Ghosh presents an upper-bound solution for bearing capacity of shallow strip footing considering composite failure mechanisms by the pseudodynamic approach. In this study, the authors used the hybrid symbiosis organisms search (HSOS) algorithm as an optimization technique and used the dynamic modules of PLAXIS-8.6v for the validation of analytical solution. The results are compared with the available literature. At the end of the study, the authors concluded that the results obtained from analytical analysis are well justified with the numerical solutions.

The study "Optimum Design of Braced Steel Space Frames including Soil-Structure Interaction via Teaching-
Learning-Based Optimization and Harmony Search Algorithms" is presented by A. T. Daloglu, M. Artar, K. Ozgan, and A. İ. Karakas. The authors used MATLAB interacting with SAP2000-OAPI codes to carry out the analysis for the 10storey braced steel space frame example. When the results obtained by using TLBO and HS are compared with each other, the total weight of the structure can be obtained more lighter with the TLBO algorithm. But the authors stated that the TLBO algorithm requires longer time for the analysis.

Another study is presented by the authors A. Kangrang, H. Prasanchum, and R. Hormwichian. Their study applied the conditional genetic algorithm (CGA) and the conditional tabu search algorithm (CTSA) technique to connect with the reservoir simulation model in order to search optimal reservoir rule curves. The results obtained from their study show that the new obtained rule curves from CTSA are more suitable for reservoir operating than the existing rule curves, and it is an effective method for application to find optimal reservoir rule curves.

An experimental study by taking into account the optimization of calcareous fly ash-added cement containing grinding aids and strength-improving additives is made by G. Kaplan, S. A. Yildizel, S. Memiş, and A. U. Öztürk. Optimization process included only the cement with fly ash and chemical additive. The main purposes of this study are the maximum level of 2-day and 28-day compressive strengths and the minimal cost parameter for the cements produced.

The study "Optimal cement mixtures containing mineral admixtures under multiple and conflicting criteria" is 
proposed by N. M. García, H. L. Soto-Toro, M. Cabrera-Ríos, and O. M. Suárez. For this multiobjective optimization problem, proper concrete bulk density, percentage of voids, and compressive strength normally are the different objective functions. Consequently, in the decision-making process, they obtained Pareto-optimal results instead of individual solution for the all objective function. They concluded that the decision makers know the best trade-off mixtures for an individual application with the help of this study.

The last study is presented by Z. Lyu, Q. Lu, Y. Song, Q. Xiang, and G. Yang. They made a case study on the design optimization of thin-walled steel-perforated sections by using artificial neural network (ANN). The main contribution of this study is to present an alternative data-driven model using ANNs to overcome some inherent difficulties associated with the design load of perforated steel members for the constructional steel field.

\section{Acknowledgments}

We thank all volunteer reviewers in the review process for this special issue. We hope that the accepted papers for special issue will lead to future works related to advanced optimization techniques.

Tayfun Dede Moacir Kripka

Vedat Toğan

Victor Yepes

Ravipudi V. Rao 


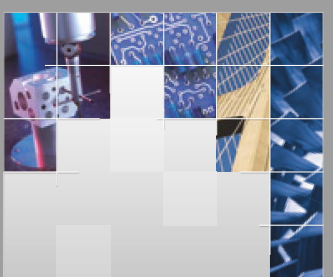

\section{Enfincering}
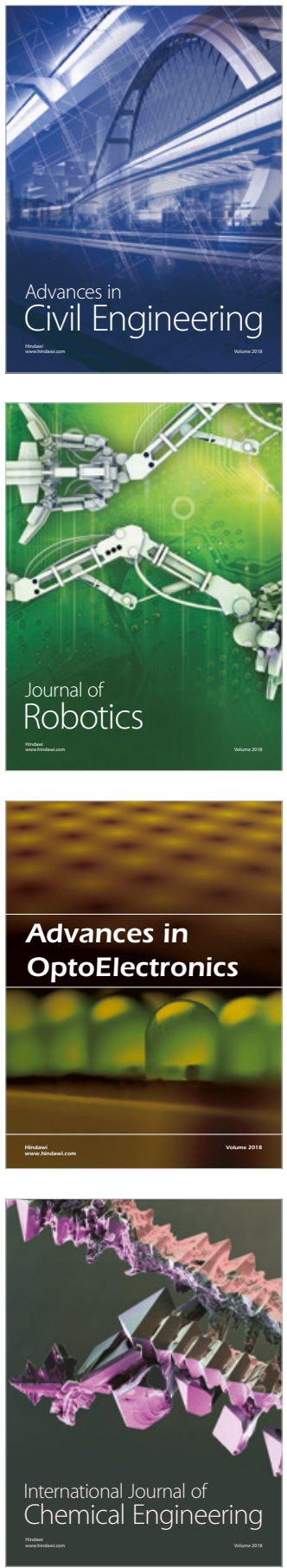

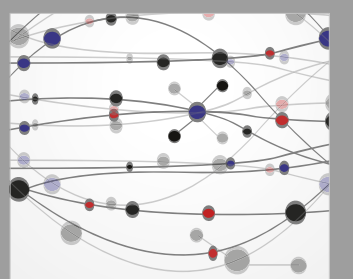

\section{Rotating \\ Machinery}

The Scientific World Journal

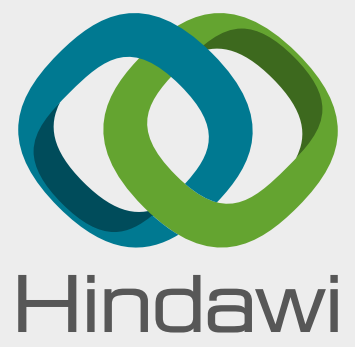

Submit your manuscripts at

www.hindawi.com
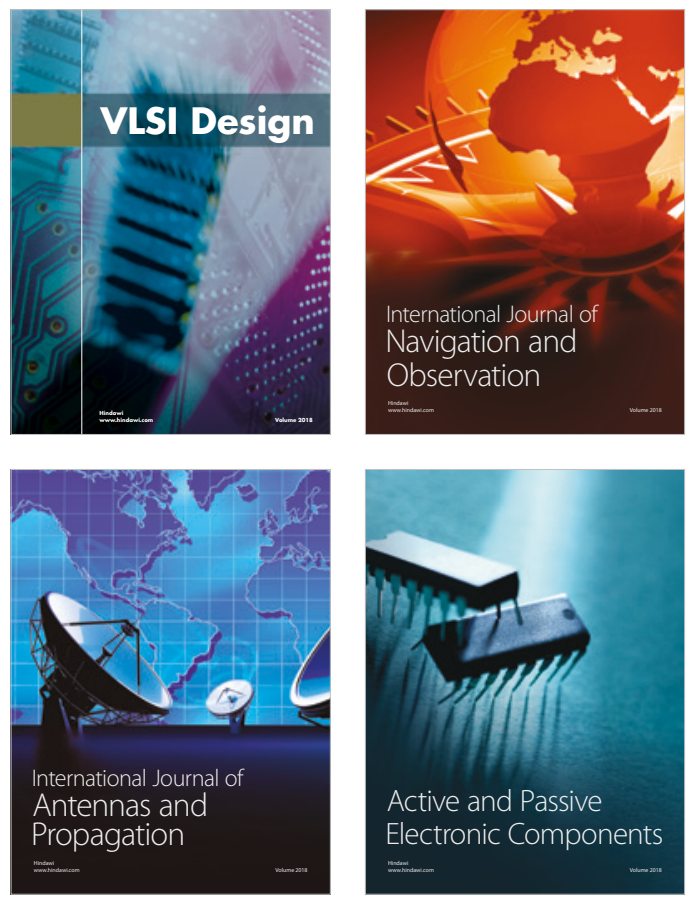
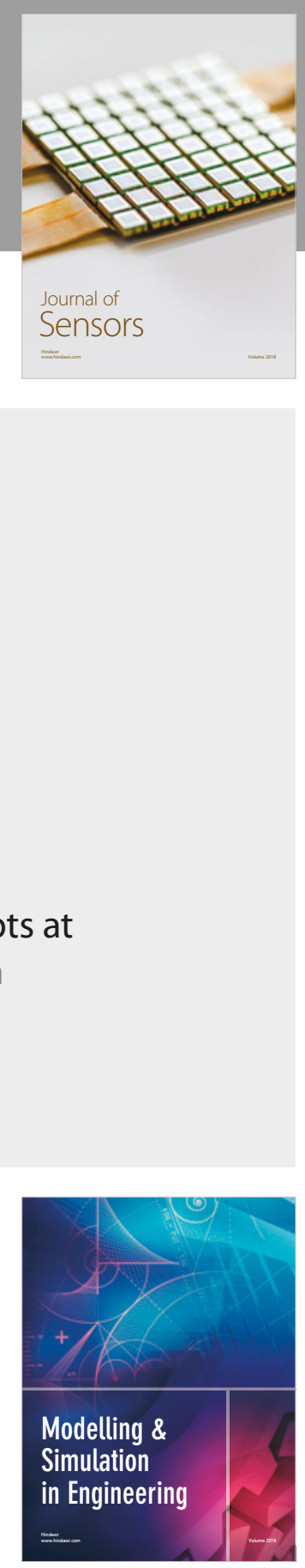

\section{Advances \\ Multimedia}
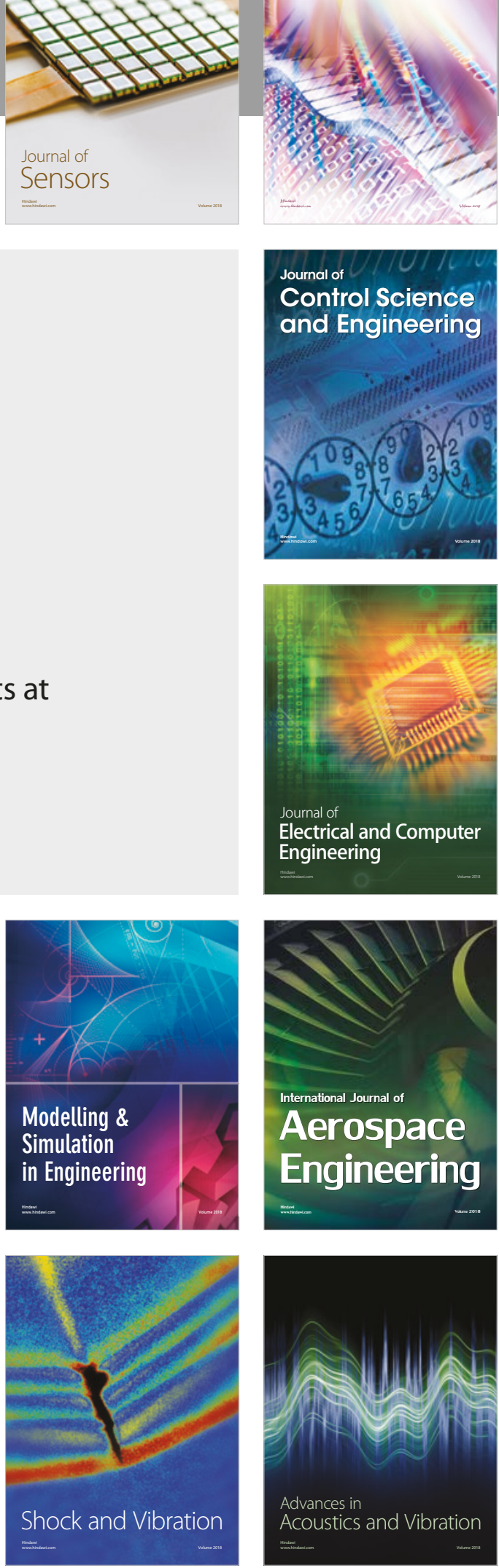